\title{
Sciendo
}

ISSN: $1231-4005$

e-ISSN: $2354-0133$

DOI: $10.2478 /$ kones-2019-0111

\section{ANALYSIS OF THE VEHICLE EXPLOITATION PROCESS WITH REGARD TO PROFILING}

\author{
Mirosław Siergiejczyk, Adam Rosiński \\ Warsaw University of Technology, Faculty of Transport \\ Koszykowa Street 75, 00-662 Warsaw, Poland \\ tel.: +48222347040, +48222341431 \\ e-mail:msi@wt.pw.edu.pl,adro@wt.pw.edu.pl
}

\begin{abstract}
Transport telematics systems integrate information technology with telecommunications for their use in various transport systems. Thanks to the use of advanced technical solutions and modern telecommunications and IT systems, it is possible to implement additional innovative services. They can be used to rationalize the process of using and maintaining means of transport. Modern vehicles are equipped with telematic on-board systems. Such solutions combine various electronic devices used in the vehicle. For this purpose, bus solutions are used, thanks to which it is possible to control individual systems and devices. It is also possible to use the transmitted data to detect negative exploitation phenomena during vehicle use (e.g. glazing phenomenon, work in the upper engine rev range with too low coolant temperature, intensive work compressor of the high air pressure system being a symptom of inability of pneumatic systems). This is possible because the data being sent has a specific information resource. Thanks to this, to can be concluded about the loss of the exploitation potential of the vehicle. This approach will be used to rationalize the technical service of the vehicle fleet, with regard to profiling. Using exploitation data, received via a telematic interface from vehicles, it is possible to profile the rolling stock. It consists in distinguishing individual sets of vehicles due to certain reliability and exploitation properties. This approach allows for the specification of the rolling stock exploitation, giving the opportunity to rationalize the use and exploitation. The publication presents the author's graph of the exploitation process taking into account the profiling of the vehicle fleet. The application of the presented approach will contribute to the improvement of the value of certain vehicle reliability and exploitation indicators.
\end{abstract}

Keywords: transport telematics systems, exploitation, reliability

\section{Introduction}

Transport telematics systems integrate information technology with telecommunications in order to use them in various transport systems. Thanks to the use of advanced technical solutions, as well as modern telecommunications and IT systems, it is possible to implement additional innovative services that can be applied to rationalise the process of using and maintaining means of transport $[12,16]$. Therefore, the efficiency of using the transport infrastructure and means of transport improves. At the same time, the safety level of travellers, vehicles and transport objects implementing the transport tasks increases [10, 14, 15]. The application of specialised IT applications in the transport area resulted in the implementation of Intelligent Transport Systems (ITS). The solutions of this type use data obtained from transport telematics systems. ITS can now be included in the group of the most advanced systems [23].

The implementation of the modern intelligent transport systems results in an increase in the safety level and timeliness of road traffic transport. The means of road transport used in the transport process should be characterised by certain values of reliability and operation indicators $[5,22]$. The reliability of vehicles is affected by the reliability of components and the applied redundant structures $[2,4]$. The first solution prevents damage. The second solution makes it possible to tolerate the occurring damage by the distinction of partial fitness. When analysing the solutions applied in the vehicles, the quality of information obtained by systems from sensors is crucial [25]. Their functioning is also affected by oscillations [3]. It is also important to meet the 
requirements in terms of electromagnetic compatibility of individual subsystems and correct power supply of electronic devices used in the vehicle [19, 24].

Modern vehicles are equipped with telematics on-board systems. Such solutions combine various electronic devices used in the vehicle. For this purpose, bus solutions are used, thanks to which it is possible to control individual systems and devices. It is also possible to use the transmitted data to detect negative operation phenomena during vehicle use (e.g. glazing phenomenon, work in the upper engine rev range with too low coolant temperature, intensive work compressor of the high air pressure system being a symptom of inability of pneumatic systems). This is possible because the data being sent have a specific information resource. Thanks to this, the loss of the operation potential of the vehicle can be concluded. This approach, with regard to profiling, will be used to rationalise the technical service of the vehicle fleet.

\section{The problem of modelling the operation process of means of transport}

In terms of maintenance activities, it is possible to distinguish two groups' preventive maintenance and corrective maintenance [18].

The preventive maintenance is aimed at the preventive maintenance performance. As a result of these activities, there is a decrease in the probability of damage or deterioration of the vehicle functioning. In the scope of activities related to the preventive maintenance, it is possible to distinguish two groups: condition based maintenance and predetermined maintenance.

In order to implement the maintenance strategy based on the condition, it is necessary to monitor the vehicle condition and then its maintenance is performed when the likelihood of losing the state of fitness occurs. During the predetermined maintenance strategy implementation, the preventive maintenance at the predetermined times (periods between individual maintenance are determined as a result of the reliability analysis or/and operation experience) are carried out.

The corrective maintenance is carried out after the vehicle damage occurrence. Then, the activities in the form of a repair restoring the state of fitness are undertaken. In the corrective maintenance, two groups can be distinguished. The first one includes immediate corrective maintenance during which the repair takes place immediately after the damage detection. The second one includes deferred corrective maintenance during which the repair is delayed in accordance with the accepted rules of conduct.

The use of modern solutions in the field of transport telematics systems and ITS in transport results in a significant increase in the amount of operational information. They can be used in order to rationalise the vehicle operation process. Such an approach can be defined by the term "maintenance". It is a concept of maintaining the vehicles, which are monitored and managed with the use of means of transport telematics. Therefore, the current assessment of degradation of vehicles is possible. At the same time, it is possible to take action to counteract adverse changes in their technical condition.

By analysing the development of the concept of maintaining means of transport, it can be concluded that at the beginning it was an approach in the form of traditional "fail and fix" maintenance practices. Then, a proactive solution, which can be described with the "predict and prevent" term, was used [9].

The development of ICT systems applied in ITS resulted in a change of the approach in management and operation of vehicles. It resulted in the creation of PHM (Prognostics and Health Management/Monitoring) systems $[1,11,17]$. The solutions of this type are characterised by the use of forecasting tools in order to manage the usability potential of technical objects (in particular, means of transport). The implementation of the PHM systems is aimed at predicting future operational events, means of transport, the usability potential assessment and RUL (Remaining Useful Life). In order to make it feasible, it is necessary to use diagnostic information. It allows determining the current state of the object, and at the same time, it is also possible to forecast the past operational states. The PHM systems were implemented in air transport at the 
earliest. Currently, they can be more often found in other transport modes (especially, rail transport) $[6,13,21]$.

In this article, the authors plan to use diagnostic information obtained from vehicles through the complex telematics systems. Such an approach allows for access to data from many sensors constituting the equipment of means of transport. This information can be then analysed with the use, among others, of cloud computing [20], artificial neural networks [7] and fuzzy logic.

\section{Modelling of the operation process of vehicles allowing for profiling}

Figure 1 presents the model of the vehicle operation process including the possibility of profiling. The object staying in the state $S_{1}$ is fit and it can implement transport tasks. In this state, the object is monitored, and the diagnosis is formulated. The positive diagnosis allows continuing the use of the object.

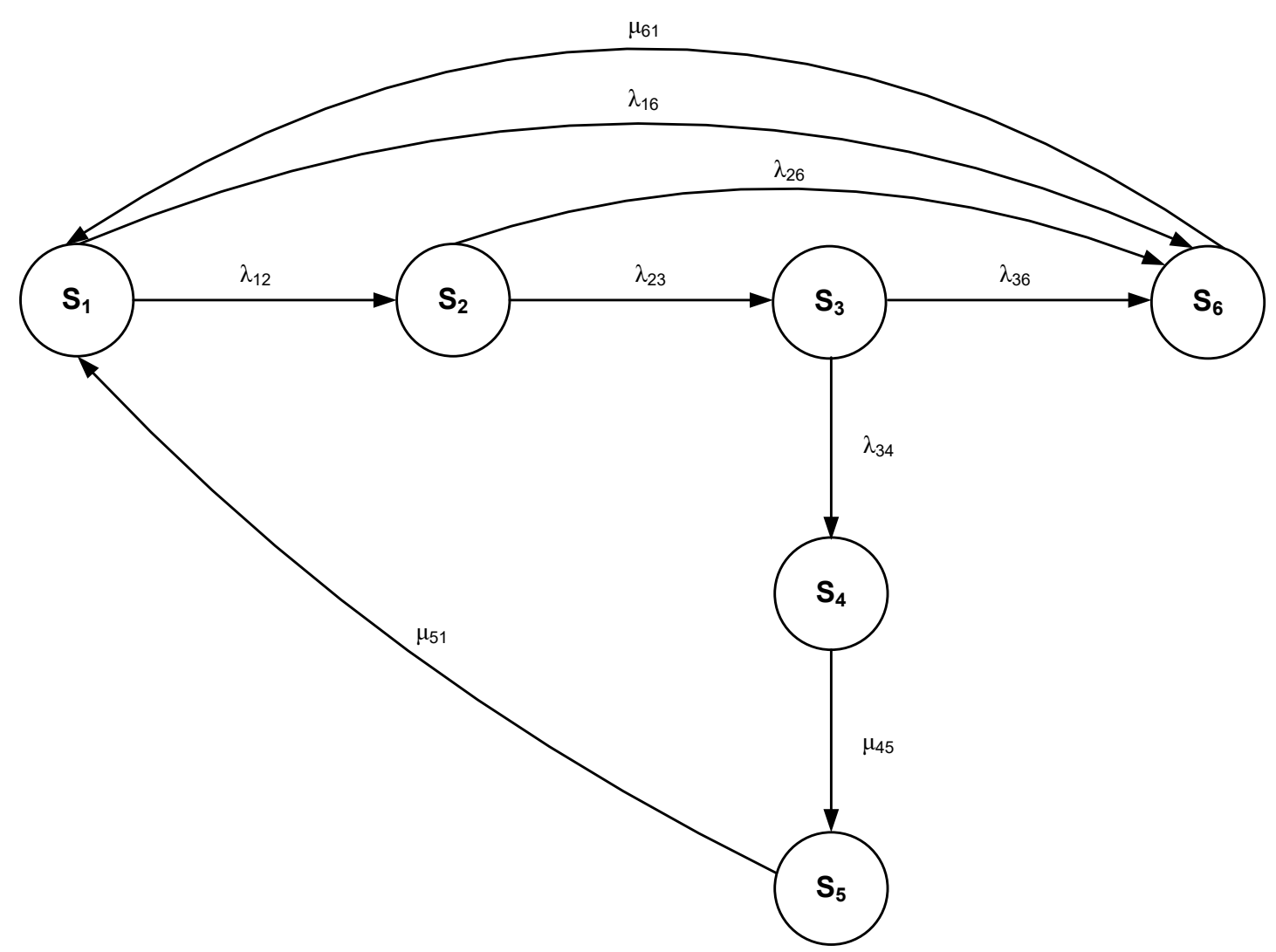

Fig. 1. Model of the fleet operation process allowing for monitoring

If the object is in the state $\mathrm{S}_{1}$ and the vehicle degradation occurs, it is transferred with the intensity $\lambda_{12}$ to the state of partial fitness $1 \mathrm{~S}_{2}$. In case of the occurrence of the further vehicle degradation process with the intensity $\lambda_{23}$, it goes to state of partial fitness 2 marked with the symbol $\mathrm{S}_{3}$. Then, with the intensity $\lambda_{34}$, it goes to the state of diagnosis $\mathrm{S}_{4}$, from which it goes to the state of maintenance $S_{5}$ with the diagnosis formulation intensity $\mu_{45}$.

Being in the state $\mathrm{S}_{5}$, it is subject to the maintenance process, and then, with the preventive maintenance intensity $\mu_{51}$, it goes to the state of fitness $S_{1}$.

It is possible for the object to go from the state of fitness $S_{1}$ to the state of unfitness $S_{6}$ and from the state $S_{2}$ to this state of unfitness $S_{6}$. Such a situation takes place in case of the occurrence of sudden (catastrophic) damage with the intensities $\lambda_{16}$ and $\lambda_{26}$. The occurrence of this type of damage can take place, when the object stays in the state $S_{3}$. Then, with the damage intensity $\lambda_{36}$, it goes to the state $\mathrm{S}_{6}$. 
The object staying in the state $\mathrm{S}_{6}$ is subject to corrective maintenance and after the repair; it is transferred with the intensity $\mu_{61}$ to the state of fitness $S_{1}$. Then, it can start the transport task implementation.

A characteristic feature of this model is the fact that the object can be used not only when it is in the state of fitness, but when it is also in the state of partial fitness 1 . In the event of degradation and transition to the state of partial fitness 1 , it performs the tasks that are feasible with a reduced usability potential of the object.

In the state of partial fitness 1 marked with the symbol $\mathrm{S}_{3}$, it is possible to profile the rolling stock by adjusting the held usability potential to the possibility of performing specific transport tasks.

Another degradation causes the loss of the usability potential to such an extent that it prevents further use of the vehicle. Then, it is subject to the preventive maintenance, previously passing through the state of diagnosis, which allows determining the scope of necessary maintenance and repairing activities.

Markings in Fig. 1:

$\mathrm{S}_{1}$ - state of fitness (complete utility potential),

$\mathrm{S}_{2}$ - state of partial fitness 1 (reduced usability potential),

$\mathrm{S}_{3}$ - state of partial fitness 2 (requiring the usability potential restoration),

$\mathrm{S}_{4}$ - state of diagnosis,

$\mathrm{S}_{5}$ - state of maintenance,

$\mathrm{S}_{6}$ - state of unfitness.

The operation process model presented in Fig. 1 can be described with the following ChapmanKolmogorov equations:

$$
\begin{aligned}
& S_{1}^{\prime}(t)=-\lambda_{16} \cdot S_{1}(t)+\mu_{61} \cdot S_{6}(t)-\lambda_{12} \cdot S_{1}(t)+\mu_{51} \cdot S_{5}(t), \\
& S_{2}^{\prime}(t)=\lambda_{12} \cdot S_{1}(t)-\lambda_{26} \cdot S_{2}(t)-\lambda_{23} \cdot S_{2}(t), \\
& S_{3}^{\prime}(t)=\lambda_{23} \cdot S_{2}(t)-\lambda_{36} \cdot S_{3}(t)-\lambda_{34} \cdot S_{3}(t), \\
& S_{4}^{\prime}(t)=-\mu_{45} \cdot S_{4}(t)+\lambda_{34} \cdot S_{3}(t), \\
& S_{5}^{\prime}(t)=-\mu_{51} \cdot S_{5}(t)+\mu_{45} \cdot S_{4}(t), \\
& S_{6}^{\prime}(t)=\lambda_{16} \cdot S_{1}(t)+\lambda_{26} \cdot S_{2}(t)+\lambda_{36} \cdot S_{3}(t)-\mu_{61} \cdot S_{6}(t)
\end{aligned}
$$

Assuming the baseline conditions:

$$
\begin{aligned}
& \mathrm{S}_{1}(0)=1, \\
& \mathrm{~S}_{2}(0)=\mathrm{S}_{3}(0)=\mathrm{S}_{4}(0)=\mathrm{S}_{5}(0)=\mathrm{S}_{6}(0)=0
\end{aligned}
$$

and applying the Laplace transform, it is possible to obtain the following system of equations:

$$
\begin{aligned}
& \left(\mathrm{s}+\lambda_{16}+\lambda_{12}\right) \cdot S_{1}^{*}(s)-\mu_{61} \cdot S_{6}^{*}(s)-\mu_{51} \cdot S_{5}^{*}(s)=1, \\
& \left(s+\lambda_{26}+\lambda_{23}\right) \cdot S_{2}^{*}(s)-\lambda_{12} \cdot S_{1}^{*}(s)=0, \\
& \left(s+\lambda_{36}+\lambda_{34}\right) \cdot S_{3}^{*}(s)-\lambda_{23} \cdot S_{2}^{*}(s)=0, \\
& \left(s+\mu_{45}\right) \cdot S_{4}^{*}(s)-\lambda_{34} \cdot S_{3}^{*}(s)=0, \\
& \left(s+\mu_{51}\right) \cdot S_{5}^{*}(s)-\mu_{45} \cdot S_{4}^{*}(s)=0, \\
& \left(s+\mu_{61}\right) \cdot S_{6}^{*}(s)-\lambda_{16} \cdot S_{1}^{*}(s)-\lambda_{26} \cdot S_{2}^{*}(s)-\lambda_{36} \cdot S_{3}^{*}(s)=0 .
\end{aligned}
$$

The probabilities of the system staying in the distinguished functional states from the symbolic (Laplace's) perspective have the following form: 


$$
\begin{aligned}
& S_{1}^{*}(s)=-\frac{b \cdot c \cdot d \cdot e \cdot f}{b \cdot c \cdot d \cdot e \cdot \lambda_{16} \cdot \mu_{61}+c \cdot d \cdot e \cdot \lambda_{12} \cdot \lambda_{26} \cdot \mu_{61}+d \cdot e \cdot \lambda_{12} \cdot \lambda_{23} \cdot \mu_{61} \cdot \lambda_{36}+}, \\
& +\mathrm{f} \cdot \lambda_{12} \cdot \lambda_{23} \cdot \lambda_{34} \cdot \mu_{51} \cdot \mu_{45}-\mathrm{a} \cdot \mathrm{b} \cdot \mathrm{c} \cdot \mathrm{d} \cdot \mathrm{e} \cdot \mathrm{f} \\
& \mathrm{S}_{2}^{*}(\mathrm{~s})=-\frac{\mathrm{c} \cdot \mathrm{d} \cdot \mathrm{e} \cdot \mathrm{f} \cdot \lambda_{12}}{\mathrm{~b} \cdot \mathrm{c} \cdot \mathrm{d} \cdot \mathrm{e} \cdot \lambda_{16} \cdot \mu_{61}+\mathrm{c} \cdot \mathrm{d} \cdot \mathrm{e} \cdot \lambda_{12} \cdot \lambda_{26} \cdot \mu_{61}+\mathrm{d} \cdot \mathrm{e} \cdot \lambda_{12} \cdot \lambda_{23} \cdot \mu_{61} \cdot \lambda_{36}+}, \\
& +\mathrm{f} \cdot \lambda_{12} \cdot \lambda_{23} \cdot \lambda_{34} \cdot \mu_{51} \cdot \mu_{45}-\mathrm{a} \cdot \mathrm{b} \cdot \mathrm{c} \cdot \mathrm{d} \cdot \mathrm{e} \cdot \mathrm{f} \\
& \mathrm{S}_{3}^{*}(\mathrm{~s})=-\frac{\mathrm{d} \cdot \mathrm{e} \cdot \mathrm{f} \cdot \lambda_{12} \cdot \lambda_{23}}{\mathrm{~b} \cdot \mathrm{c} \cdot \mathrm{d} \cdot \mathrm{e} \cdot \lambda_{16} \cdot \mu_{61}+\mathrm{c} \cdot \mathrm{d} \cdot \mathrm{e} \cdot \lambda_{12} \cdot \lambda_{26} \cdot \mu_{61}+\mathrm{d} \cdot \mathrm{e} \cdot \lambda_{12} \cdot \lambda_{23} \cdot \mu_{61} \cdot \lambda_{36}+}, \\
& +\mathrm{f} \cdot \lambda_{12} \cdot \lambda_{23} \cdot \lambda_{34} \cdot \mu_{51} \cdot \mu_{45}-\mathrm{a} \cdot \mathrm{b} \cdot \mathrm{c} \cdot \mathrm{d} \cdot \mathrm{e} \cdot \mathrm{f} \\
& \mathrm{S}_{4}^{*}(\mathrm{~s})=-\frac{\mathrm{e} \cdot \mathrm{f} \cdot \lambda_{12} \cdot \lambda_{23} \cdot \lambda_{34}}{\mathrm{~b} \cdot \mathrm{c} \cdot \mathrm{d} \cdot \mathrm{e} \cdot \lambda_{16} \cdot \mu_{61}+\mathrm{c} \cdot \mathrm{d} \cdot \mathrm{e} \cdot \lambda_{12} \cdot \lambda_{26} \cdot \mu_{61}+\mathrm{d} \cdot \mathrm{e} \cdot \lambda_{12} \cdot \lambda_{23} \cdot \mu_{61} \cdot \lambda_{36}+}, \\
& +\mathrm{f} \cdot \lambda_{12} \cdot \lambda_{23} \cdot \lambda_{34} \cdot \mu_{51} \cdot \mu_{45}-\mathrm{a} \cdot \mathrm{b} \cdot \mathrm{c} \cdot \mathrm{d} \cdot \mathrm{e} \cdot \mathrm{f} \\
& S_{5}^{*}(s)=-\frac{f \cdot \lambda_{12} \cdot \lambda_{23} \cdot \lambda_{34} \cdot \mu_{45}}{b \cdot c \cdot d \cdot e \cdot \lambda_{16} \cdot \mu_{61}+c \cdot d \cdot e \cdot \lambda_{12} \cdot \lambda_{26} \cdot \mu_{61}+d \cdot e \cdot \lambda_{12} \cdot \lambda_{23} \cdot \mu_{61} \cdot \lambda_{36}+}, \\
& +\mathrm{f} \cdot \lambda_{12} \cdot \lambda_{23} \cdot \lambda_{34} \cdot \mu_{51} \cdot \mu_{45}-\mathrm{a} \cdot \mathrm{b} \cdot \mathrm{c} \cdot \mathrm{d} \cdot \mathrm{e} \cdot \mathrm{f} \\
& \mathrm{S}_{6}^{*}(\mathrm{~s})=-\frac{\mathrm{c} \cdot \mathrm{d} \cdot \mathrm{e} \cdot \lambda_{12} \cdot \lambda_{26}+\mathrm{d} \cdot \mathrm{e} \cdot \lambda_{12} \cdot \lambda_{23} \cdot \lambda_{36}+\mathrm{b} \cdot \mathrm{c} \cdot \mathrm{d} \cdot \mathrm{e} \cdot \lambda_{16}}{\mathrm{~b} \cdot \mathrm{c} \cdot \mathrm{d} \cdot \mathrm{e} \cdot \lambda_{16} \cdot \mu_{61}+\mathrm{c} \cdot \mathrm{d} \cdot \mathrm{e} \cdot \lambda_{12} \cdot \lambda_{26} \cdot \mu_{61}+\mathrm{d} \cdot \mathrm{e} \cdot \lambda_{12} \cdot \lambda_{23} \cdot \mu_{61} \cdot \lambda_{36}+}, \\
& +\mathrm{f} \cdot \lambda_{12} \cdot \lambda_{23} \cdot \lambda_{34} \cdot \mu_{51} \cdot \mu_{45}-\mathrm{a} \cdot \mathrm{b} \cdot \mathrm{c} \cdot \mathrm{d} \cdot \mathrm{e} \cdot \mathrm{f}
\end{aligned}
$$

where:

$$
\begin{aligned}
& \mathrm{a}=\mathrm{s}+\lambda_{16}+\lambda_{12}, \\
& \mathrm{~b}=\mathrm{s}+\lambda_{26}+\lambda_{23}, \\
& \mathrm{c}=\mathrm{s}+\lambda_{36}+\lambda_{34}, \\
& \mathrm{~d}=\mathrm{s}+\mu_{45}, \\
& \mathrm{e}=\mathrm{s}+\mu_{51}, \\
& \mathrm{f}=\mathrm{s}+\mu_{61} .
\end{aligned}
$$

The solution of a set of equations (4) in the field of time is the next stage of the analysis and it is not implemented in this article.

By conducting the further mathematical analysis of the set of equations (4), the relationships that allow determining the probabilities of the system staying in the distinguished states are obtained.

\section{Conclusions}

The article presents the author's graph of the operation process including the possibility of profiling the vehicle fleet. By using the operation data obtained by the telematic interface from the vehicles, it is possible to profile the rolling stock. It involves the distinction of individual sets 
of vehicles in terms of specific reliability and operation properties. Such an approach allows for the rolling stock operation specification that provides the opportunity to rationalise the operation and maintenance. The application of the presented approach will contribute to the improvement of the value of certain vehicle reliability and operation indicators.

In further studies of this area, the authors plan to use operation data received via telematics interface from the vehicle in order to determine the Remaining Useful Life. It should contribute to the further improvement of the value of reliability and operation indicators of the vehicle fleet.

\section{References}

[1] Atamuradov, V., Medjaher, K., Dersin, P., Lamoureux, B., Zerhouni, N., Prognostics and health management for maintenance practitioners - review, implementation and tools evaluation, International Journal of Prognostics and Health Management, Vol. 8 (31), pp. 1-31, 2017.

[2] Będkowski, L., Dąbrowski, T., The basis of exploitation, part II: The basis of exploitation reliability, Military University of Technology, Warsaw 2006.

[3] Burdzik, R., Konieczny, Ł., Figlus, T., Concept of on-board comfort vibration monitoring system for vehicles, Activities of Transport Telematics, TST 2013, J. Mikulski (Ed.), Vol. 395, pp. 418-425, Springer, Heidelberg 2013.

[4] Caban, D., Walkowiak, T., Dependability analysis of hierarchically composed system-ofsystems, Proceedings of the Thirteenth International Conference on Dependability and Complex Systems DepCoS-RELCOMEX, Zamojski W., Mazurkiewicz J., Sugier J., Walkowiak T., Kacprzyk J. (Eds.), pp. 113-120, Springer, 2019.

[5] Chrzan, M., Kornaszewski, M., Ciszewski, T., Renovation of marine telematics objects in the process of exploitation, Management Perspective for Transport Telematics, TST 2018, Communications in Computer and Information Science, J. Mikulski (Ed.), Vol. 897, Springer, Cham 2018.

[6] Chudzikiewicz, A., Bogacz, R., Kostrzewski, M., Konowrocki, R., Condition monitoring of railway track systems by using acceleration signals on wheelset axle-boxes, Transport, Vol. 33(2), pp. 555-566, 2018.

[7] Duer, S., Duer, R., Mazuru, S., Determination of the expert knowledge base on the basis of a functional and diagnostic analysis of a technical object, Romanian Association of Nonconventional Technologies, Romanian, 6/2016, Vol. XX, No. 2, pp. 23-29, 2016.

[8] Frei, M., Automotive data buses in workshop practice, WKŁ, Warsaw 2016.

[9] Galar, D., Wandt, K., Karim, R., Berges, L., The evolution from e(lectronic) Maintenance to i(ntelligent) Maintenance, Insight-Non-Destructive Testing and Condition Monitoring, Vol. 54 (8), pp. 1-6, 2012.

[10] Janusova, L., Cicmancova, S., Improving safety of transportation by using inteligent transportation systems, 9th International Scientific Conference Transbaltica 2015, Procedia Engineering, Vol. 134, pp. 14-22, 2016.

[11] Jia, X., Huang, B., Feng, J., Cai, H., Lee, J., A review of PHM Data Competitions from 2008 to 2017: Methodologies and Analytics, Proceedings of the Annual Conference of the Prognostics and Health Management Society 2018, pp. 1-10, Philadelphia 2018.

[12] Kasprzyk, Z., Rychlicki, M., Analysis of physical layer model of WLAN 802.11g data transmission protocol in wireless networks used by telematic systems, Proceedings of the Ninth International Conference on Dependability and Complex Systems DepCoSRELCOMEX, Advances in Intelligent Systems and Computing, Vol. 286, pp. 265-274, Springer International Publishing, 2014.

[13] Kour, R., Karim, R., Tretten, P., eMaintenance solutions for railway maintenance decisions, Proceedings of the World Congress on Engineering, Vol. 1, London 2014.

[14] Lewiński, A., Perzyński, T., Toruń, A., The analysis of open transmission standards in 
railway control and management, Telematics in the Transport Environment, TST 2012, Communications in Computer and Information Science, Vol. 329, pp. 10-17, SpringerVerlag, Berlin Heidelberg 2012.

[15] Losurdo, F., Dileo, I., Siergiejczyk, M., Krzykowska, K., Krzykowski, M., Innovation in the ICT infrastructure as a key factor in enhancing road safety: a multi-sectoral approach, Proceedings 25th International Conference on Systems Engineering ICSEng 2017, H. Selvaraj, G. Chmaj, D. Zydek (Eds.), IEEE Computer Society Conference Publishing Services (CPS), pp. 157-162, Las Vegas 2017.

[16] Łubkowski, P., Laskowski, D., Selected issues of reliable identification of object in transport systems using video monitoring services, Communication in Computer and Information Science, J. Mikulski (Ed.), Vol. 471, pp. 59-68, Springer, Berlin Heidelberg 2015.

[17] Mesgarpour, M., Landa-Silva, D., Dickinson, I., Overview of telematics-based prognostics and health management systems for commercial vehicles, Communications in Computer and Information Science, vol. 395, Activities of Transport Telematics, TST 2013, J. Mikulski (Ed.), pp. 123-130, Springer-Verlag, Berlin Heidelberg 2013.

[18] Nowakowski, T., Tubis, A., Werblińska-Wojciechowska, S., Evolution of technical maintenance approaches - review and a case study, Intelligent Systems in Production and Maintenance, ISPEM 2018, Advances in Intelligent Systems and Computing, Vol. 835, pp. 161-174, Springer, Cham 2019.

[19] Paś, J., Operation of electronic transportation systems, Publishing House University of Technology and Humanities, Radom 2015.

[20] Reddig, K., Dikunow, B., Krzykowska, K., Proposal of big data route selection methods for autonomous vehicles, Internet Technology Letters, Vol. 1 (36), pp. 1-6, 2018.

[21] Restel, F. J., The Markov reliability and safety model of the railway transportation system, Safety and Reliability: Methodology and Applications - Proceedings of the European Safety and Reliability Conference, ESREL 2014, pp. 303-311.

[22] Rosiński, A., Modelling the maintenance process of transport telematics systems, Publishing House Warsaw University of Technology, Warsaw 2015.

[23] Siergiejczyk, M., Krzykowska, K., Rosiński, A., Grieco, L. A., Reliability and viewpoints of selected ITS system, Proceedings 25th International Conference on Systems Engineering ICSEng 2017, H. Selvaraj, G. Chmaj, D. Zydek (Eds.), IEEE Computer Society Conference Publishing Services (CPS), pp. 141-146, Las Vegas 2017.

[24] Siergiejczyk, M., Paś, J., Rosiński, A., Modeling of process of maintenance of transport systems telematics with regard to electromagnetic interferences, Tools of Transport Telematics, Mikulski J. (Ed.), pp. 99-107, Springer-Verlag, Berlin Heidelberg 2015.

[25] Stawowy, M., Kasprzyk, Z., Identifying and simulation of status of an ICT system using rough sets, Proceedings of the Tenth International Conference on Dependability and Complex Systems DepCoS-RELCOMEX, Advances in intelligent systems and computing, Vol. 365, pp. 477-484, Springer, 2015.

Manuscript received 08 July; approved for printing 22 November 2019 